\section{The 37th Annual Meeting of the J.B. Johnston Club for Evolutionary Neuroscience and the 29th Annual Karger Workshop in Evolutionary Neuroscience}

The 2017 meetings of the J.B. Johnston Club for Evolutionary Neuroscience and Karger Workshop in Evolutionary Neuroscience will be held immediately before the annual meeting of the Society for Neuroscience on Thursday, November 9 (the Karger Workshop), and Friday, November 10 (the regular JBJC meeting). Both meetings will take place at the College Park Marriott in Hyattsville, MD, USA.

This year's Karger Workshop, made possible by the continuing support of Karger Publishers, is organized by Ashley Morhardt. It is titled "From Fossils to Function: Integrative and Diverse Approaches to Vertebrate Evolutionary Neuroscience." The Workshop features seven invited talks by speakers that will attempt to elucidate the evolution of the vertebrate brain through comparative studies in a wide array of extant and extinct vertebrate taxa, with special focus on groundbreaking structural and functional neuroimaging techniques. On the following day, the program for the annual JBJC meeting will consist of 19 talks submitted by JBJC members selected by the JBJC Program Committee (Naoyuki Yamamoto, Kara Yopak, and Paul Forlano) plus a presentation by this year's invited Karger Speaker, Dr. David Van Essen. The meeting also includes a "data blitz" of very brief presentations from graduate students and postdoctoral researchers. Additional information and the final schedule of talks will be mailed to JBJC members before the meeting and posted on the JBJC web site (www. jbjclub.ning.com).

\section{Karger Workshop in Evolutionary Neuroscience: From Fossils to Function: Integrative and Diverse Approaches to Vertebrate Evolutionary Neuroscience}

Speakers giving presentations at the 2017 Karger Workshop in Evolutionary Neuroscience are listed below. The final schedule of talks will be sent to the membership prior to the meeting and will be available at the registration desk during the meeting.

- Andrew Iwaniuk, University of Lethbridge, Lethbridge, AB, Canada
- Amy Balanoff, Johns Hopkins School of Medicine, Baltimore, MD, USA

- Emiliano Bruner, Centro Nacional de Investigación sobre la Evolución Humana, Burgos, Spain

- Haley O’Brien, Oklahoma State University Center for Health Sciences, Tulsa, OK, USA

- Paul Gignac, Oklahoma State University Center for Health Sciences, Tulsa, OK, USA

- James Rilling, Emory University, Atlanta, GA, USA

- David Van Essen, Washington University School of Medicine in St. Louis, St. Louis, MO, USA

\section{J.B. Johnston Club for Evolutionary Neuroscience Meeting Abstracts}

Abstracts for talks scheduled for the 2017 annual meeting of the J.B. Johnston Club for Evolutionary Neuroscience are listed in alphabetical order by presenting author. The final schedule of talks will be sent to the membership prior to the meeting and will be available at the registration desk during the meeting. This year's Karger Invited Guest will be Dr. David Van Essen. The title of his talk will be: "Parcellation of Cerebral Cortex in Humans and NonHuman Primates.”

\section{Duplicitous Retina: Development Suggests that Rods Evolved from UV-Sensitive Cones \\ Allison WT ${ }^{a-c}$, Oel APa \\ aDepartment of Biological Sciences, b Department of Medical Genetics, and ${ }^{\mathrm{C} C e n t r e}$ for Prions \& Protein Folding Diseases, University of Alberta, Edmonton, AB, Canada ted.allison@ualberta.ca}

The duplex retina has been a critical element of vertebrates' success, because rods permit dim light vision, whereas cones enable moderate- and bright-light vision, colour- and high acuityvision. Most vertebrates have rods and four distinct classes of cones, though this complexity has been lost in mammals. The evolutionary history of rod and cone photoreceptors has yet to be revealed. Disparate lines of evidence have led us to the novel hypoth-

\section{KARGER}

(C) 2017 S. Karger AG, Basel 
esis that rods may have evolved from short-wavelength-sensitive cones, which are maximally sensitive to ultraviolet (UV) light in most extant vertebrates: (1) degeneration of UV cones in trout is accompanied by an increase in rods; (2) mutation of disparate transcription factors in both mice and zebrafish lead to an apparent conversion of rods to UV cones. Here we synthesize literature and provide new data, revealing a suite of unexpected similarities exists between rods and UV cones across vertebrate development, morphology, physiology and evolution. Conversion of UV cones to rods appears to have occurred in multiple phases of evolution, including perhaps prior to evolution of jawed vertebrates and during emergence of mammals via the nocturnal bottleneck.

\section{Specification of Male versus Female Acoustic Communication Behaviors in Drosophila virilis}

Baker CA, Guan X-J, Fletcher M, Murthy M

Princeton Neuroscience Institute, Princeton University,

Princeton, NJ, USA

christab@princeton.edu

How brains integrate sensory information to influence behavior is a central question in neuroscience. A particularly interesting behavior for studying this problem is acoustic duetting, in which males and females control the timing of their courtship songs relative to one another. During a duet, animals must rapidly assess sensory input from their partner to then appropriately pattern their own song. Despite the prevalence of duetting across the animal kingdom (Yoshida \& Okanoya 2005, Cognitive Studies), the underlying neural mechanisms remain largely unknown. Our lab has recently developed Drosophila virilis as a model for studying acoustic duetting. Male and female $D$. virilis produce spectrotemporally distinct songs via wing vibration; males coordinate their songs with females based on auditory feedback, while females rely largely on tactile feedback to time their songs (LaRue et al. 2015, eLife). Female song is present in many fewer Drosophila species than male song. In Drosophila melanogaster, in which only males sing, females have a latent circuit capable of producing song when artificially activated (Clyne \& Miesenboch 2008, Cell). These findings raise two important questions. First, how do male and female D. virilis song circuits produce sexually dimorphic songs, and second, what changes in neural circuitry occurred to give rise to natural female song in $D$. virilis? Addressing these questions will require developing genetic tools in $D$. virilis to access song circuits. Here we test whether the fruitless gene, which plays a critical role in directing male-specific courtship behaviors in D. melanogaster, contributes to song production in $D$. virilis.

Male courtship behaviors of $D$. melanogaster, in which only males sing, are specified by sex-specific splicing of the fruitless gene. Splicing at the male site results in expression of a male-specific protein (FruM) in a subset of neurons, whereas splicing at the female site results in RNA degradation. Optogenetic activation of the neurons expressing fruitless RNA in female D. melanogaster generates male-like song, demonstrating that females have a circuit capable of producing song even though females do not naturally sing (Clyne \& Miesenboch 2008, Cell). Further, when female D. melanogaster are forced to express FruM, they court wild-type females and sing (without artificial activation) (Demir \& Dickson 2005, Cell), indicating that FruM is sufficient to specify male courtship behaviors, including singing. If fruitless plays a similar role in the duets of $D$. virilis, it may provide genetic access to the song circuits of both sexes.

To test whether fruitless specifies male song circuits in D. virilis, we used CRISPR-Cas9 mutagenesis to force production of the male-specific FruM protein in both sexes. Antibody staining and RT-PCR confirmed that mutant female brains expressed FruM. As in D. melanogaster, FruM expression caused D. virilis females to sing male song while courting wild-type females, demonstrating that fruitless indeed specifies male song circuitry in D. virilis. FruM D. virilis females duetted with wild-type males but produced altered song parameters, suggesting potential overlap between male and female song circuits. Ongoing work is generating tools to test whether fruitless RNA-expressing neurons in females are sufficient for driving song production and to further dissect the role of fruitless-expressing neurons in acoustic duetting.

\section{The Mysteries of Magnetoreception: The Role of UV Cones in Zebrafish Cryptochrome Expression}

Balay $S^{a}$, Allison $W T^{a, b}$

${ }^{\mathrm{a}}$ Department of Biological Sciences, and ${ }^{\mathrm{b}}$ Department of Medical Genetics, University of Alberta, Edmonton, AB, Canada

sbalay@ualberta.ca

It has long been known that a variety of animals use the Earth's magnetic field as a navigational cue. However, the mechanism enabling this phenomenon remains to be elucidated. Highly conserved light sensitive proteins called Cryptochrome (Cry) are putative molecular candidates due to their biophysical properties: magnetic fields can alter their chemical products, they are activated by short wavelength light and have been shown to be associated with retinal UV cone photoreceptors in birds and the homologous blue cone photoreceptors in some mammals.

The role of UV cones in magnetoreception has mostly been inferred from immunohistological studies, showing that Cry is located in the outer segments of UV cones in birds. However, the actual requirement for these photoreceptors in magnetoreceptive signalling and behavior has yet to be tested, in part due to the inaccessibility of genetic manipulation within avian models. This project aims to break down the molecular barrier of magnetoreception by using the highly versatile fish model, zebrafish (Danio rerio). Zebrafish have four cone photoreceptor subtypes (UV, blue, green and red) and represent an excellent system for studying visuallymediated behaviors. Zebrafish also possess six cry genes, and while most participate in the timing of biological rhythms via the circadian clock, the function of two of cry genes remain unknown. We sought to test if a specific cry gene was expressed in zebrafish retinal cone photoreceptors. We hypothesized that sws 1 cones are exclusively associated with cry. Loss of $s w s 1$ cones were predicted to result in decreased expression of cry.

Using pharmacogenetic nitroreductase (NTR)-mediated cell ablation technology, UV (sws1) and blue (sws2) cone subtypes were selectively removed from zebrafish retina. Fluorescent in situ hybridization and quantitative-PCR (qPCR) were used to quantify cry 
and sws gene expression. Our preliminary work shows that zebrafish UV cones express a non-circadian cry isoform. When UV cones (sws 1) were ablated, cry expression decreased. Interestingly, when blue cones (sws2) were ablated, cry expression was unchanged, suggesting that UV cones are specifically associated with cry. This study serves as the first experimental evidence that (1) cone photoreceptors influence cry expression, (2) cry and UV cones are colocalized, and (3) both may play a role in fish magnetoreception.

Although zebrafish are a non-migratory species, this system can be used to model magnetoreception in other animals. Migratory salmonids regenerate UV cones as they prepare to migrate back to their natal streams for spawning. Currently, the functional significance of this regeneration remains unknown. UV cones may enable magnetoreception via cry expression, which could provide a novel explanation for this process, and further insight into the mysteries of magnetoreception.

\section{Mesencephalic Origin of the Inferior Lobe and the Preglomerular Nucleus in Zebrafish}

\author{
Bloch S, Thomas M, Yamamoto K \\ Paris-Saclay Institute of Neuroscience (Neuro-PSI), \\ CNRS, Université Paris-Sud, Université Paris-Saclay, \\ Gif-sur-Yvette, France \\ sccbloch@gmail.com
}

The basic organization of the brain is relatively conserved throughout vertebrates. Nonetheless, the organization within subregions such as the telencephalon and the hypothalamus is largely diversified when comparing different vertebrate groups. Notably teleosts and tetrapods belong to different groups within the Osteichthyes (bony vertebrates), and their brain organization is significantly different. Current models of the vertebrate brain organization are largely based on a mammalian-centric point of view, but comparative analyses including teleost brains provide new perspectives on the general organization of the vertebrate brains.

Thanks to the availability of transgenic lines, the zebrafish is a convenient model in order to understand the basic organization of the teleost brain. We took advantage of the tamoxifen inducible cre-loxp system to perform a cell lineage study. At 24 hours postfertilisation (hpf), the transcription factor her 5 is specifically expressed in progenitors of the midbrain hindbrain boundary (MHB). Thus by crossing the two existing transgenic lines (her5:ERT2creERT2 and ßactin:loxP-STOP-loxP-mcherry), we can trace progenies of cells originating from the MHB. We induced cre recombination at $24 \mathrm{hpf}$ by treating the zebrafish embryos with tamoxifen, and followed the mcherry-expressing cells in the developing and adult brains.

As demonstrated in a recent publication (Galant et al. 2016, Developmental Biology), mcherry expression was found in mesencephalic structures such as the optic tectum and the tegmentum. In addition, we found the mcherry-positive cells more rostrally, participating in the formation of some brain structures which have been considered to be prosencephalic (forebrain). Such structures include the inferior lobe (IL) and the preglomerular nucleus (PG).
The inferior lobe (IL) is a structure that is not found in tetrapods. It develops around the caudolateral elongation of the lateral recess (LR) and has been assumed to be the posterior part of the hypothalamus. Our data show that cells forming the majority of the IL are actually mesencephalic.

The PG has been considered to be part of the ventral diencephalon called the posterior tuberculum. It is a major sensory relay nucleus in teleosts, similarly to the thalamus in amniotes. Our data show that a ventral portion of the PG is formed by mesencephalic cells. This indicates that the similar functional properties found in the amniote thalamus and the teleost PG are convergent evolution.

These results highlight important variations in brain structures in different vertebrate groups, suggesting that the nervous system is highly plastic during the vertebrate evolution.

\section{The Vocabulary of Communication in Pulse Gymnotiforms: Jamming Avoidance and Beyond}

\author{
Braun $C B^{a}$, Alves-Gomes $J^{b}$ \\ aDepartment of Psychology, Hunter College (CUNY), \\ New York, NY, USA; 'baboratório de Fisiologia \\ Comportamental e Evolução (LFCE), Instituto Nacional de \\ Pesquisas de Amazônia (INPA), Manaus, Brazil \\ cbraun@hunter.cuny.edu
}

The power of the gymnotiform neuroethological model is rooted in the relative simplicity of the electrosensory-motor loop and the ability to experimentally probe the sensory motor transforms. An intrinsically rhythmic pacemaker nucleus drives the electric organ discharges (EOD) and a relatively small number of prepacemaker inputs modulate pacemaker output. The modulations of the pacemaker rhythm include both gradual and abrupt changes in frequency, interruptions, and high-frequency bursts known as chirps. The electric fish model was pioneered by Heiligenberg and colleagues based upon the relatively simple and nearly reflexlike shifts in frequency in response to conspecifics discharging with a similar frequency, a behavior known as the Jamming Avoidance Response (JAR). The regularity of the response in some species of Eigenmannia has been an extremely powerful tool in physiological experiments, but the majority of gymnotiform pulse fishes, however, do not show a reflexive JAR. In controlled playbacks and paired interactions, many species display a flexible repertoire of interactive shifts in EOD rate. We have identified eight specific behaviors in these interactions and propose a vocabulary of jamming responses based on the specific timing of the relatively simple pacemaker modulations listed above. These behaviors include both jamming avoidance and active attempts to interfere with a conspecific's electrolocation ability. In a dyad of pulse fishes, jamming interference occurs when multiple successive EODs occur near simultaneously. As the latency between the partners' EODs increases, interference is minimized. Jamming interference may be minimized by increasing the difference in frequency (decreasing the duration of the jamming phases of the beat cycle and fewer near coincident pulses per beat). Jamming interference can also be minimized by matching of the partners EOD rates, providing that they synchronize with a relatively long latency (non-coincident phase). 
Conversely, frequency matching may also incur jamming interference if the partners match frequencies at short latencies. The behaviors identified include smooth frequency shifts to increase the frequency difference (1: fJAR) or to decrease the frequency difference, which, depending on latency, increases jamming interference (2: fJAM) or decreases it (3: fMatch). Alternatively, some species make abrupt frequency increases at specific phases of the beat cycle, minimizing the number of coincident pulses per beat (4: burstJAR). Many species show frequency matching, sometimes very tightly at near-coincident phases (fJAM) or with ongoing modulations in the frequency difference resulting in repetitive scanning of near-coincindent phases (5: scanning). Many species also demonstrate pauses in EOD rhythm putatively caused by distinct prepacemaker inputs (6: hushing silence and 7: interruptions). Finally, many species also demonstrate brief large increases in pacemaker rhythm known as (8) chirps. We speculate that all of these behaviors are used in interactive communication over territory and status, as well as in mating contexts.

\section{Invariant Microglial Cells Densities Suggest Conserved Developmental and Evolutionary Mechanisms Governing Their Addition to Mammalian Brains}

\author{
Dos Santos $S E^{a}$, Botelho $L^{b}$, Medeiros $M^{b}$, Porfirio $J^{b}$, Messeder $D^{b}$, \\ Herculano-Houzel $S^{a, c, d}$
}

${ }^{a}$ Department of Psychology, Vanderbilt University, Nashville, TN, USA; 'Instituto de Ciências Biomédicas, Universidade Federal do Rio de Janeiro, Rio de Janeiro, Brazil; ' $D$ epartment of Biological Sciences, and dVanderbilt Brain Institute, Vanderbilt University, Nashville, TN, USA

sandra.e.dos.santos@vanderbilt.edu

Brain structure mass scales as a universal function of numbers of non-neuronal cells, but as clade- and structure-specific functions of numbers of neurons, across mammalian species. The universality of the relationship for non-neuronal cells however does not discern whether similar scaling rules apply to endothelial cells and individual glial cell subtypes.

Here we aim to determine if the universal scaling rule found for non-neuronal cells as a whole applies to microglial cells in particular. In that case, brain structure mass should scale universally with their numbers of microglial cells across species, and microglial cell densities should be found to remain invariant across brain structures and species, as found previously for non-neuronal cells as a whole.

Microglial cells are the resident macrophages of the brain whose actions contribute to the maturation and plasticity of neural circuits that ultimately shape behavior. Invariant microglial cell densities across brain structures and species would be consistent with their key role in the construction and modeling of brain structures.

We quantified the total number of microglial cells, their proportion to non-neuronal cells as a whole, and the ratio of microglial cells to neurons in species belonging to several mammalian clades. We used the isotropic fractionator and immunocytochem- istry to anti-Ibal and anti-NeuN antibodies to estimate numbers of microglial cells and neurons in several brain structures. We examined the brains of 33 species of mammals (10 marsupials, 4 artiodactyls, 8 primates, 5 afrotherians and 6 carnivores).

Microglial cells represent between $1 \%$ of all cells in the cerebellum and $12 \%$ in the cerebral cortex and rest of brain, at densities between 1,000 and 10,000 cells/mg. Each brain structure gains microglial cells as a similar power function of its number of neurons across species. Importantly, all brain structures vary in mass across all species as a single power function of the number of microglial cells in the structure with a joint exponent of $0.956 \pm 0.024(p<$ 0.0001 ) indicating that brain structures of similar size are composed of similar numbers of microglial cells across different mammalian clades. Additionally, the ratio between microglial cells and neurons decreases universally with increasing neuronal density across structures and species, as found for the ratio between numbers of other cells as a whole and of neurons.

The present finding that numbers of microglial cells scale in a similar manner across the wide range of mammals studied, and in all major brain structures, indicates that the addition of these cells to the brain is governed by conserved developmental and evolutionary mechanisms.

\section{Assessing the Utility of Avian Brain Endocasts as Predictors of Vision-Related Neuroanatomy and Potential Functional Capabilities}

\author{
Early $C M^{a}$, Ridgely $R C^{b}$, Witmer $L M^{b}$
}

aDepartment of Biological Sciences, and bepartment of Biomedical Sciences, Ohio University, Athens, $\mathrm{OH}$, USA

ce643812@ohio.edu

Avian brain endocasts are relatively faithful representations of the external morphology of the brain, and in extinct birds, they are the only source of information on brain anatomy. As such, they are often used to infer behaviors in extinct birds. However, the correlations between brain structures and behaviors in extant birds are based on volume, whereas the size metric for brain structures available from endocasts is surface area. The assumption has been that the surface area of an endocast structure is a fair proxy for the volume of the underlying brain structure, but this is problematic. First, the endocast only allows measurement of the surface area of externally-visible portions of brain structures. Additionally, a statistical relationship between surface area of an endocast structure and volume of the brain structure has not been established. The optic lobe, which overlies the optic tectum, and the Wulst, which overlies the hyperpallium, are good candidates for this study due to their presence on avian brain endocasts across a broad taxonomic sample. The volume of the retinorecipient optic tectum may correlate with visual abilities, but other structures located deep to the optic lobe may also influence its size. The hyperpallium is part of the avian thalamofugal visual pathway and is involved in higherorder visual processing as well as somatosensory and motor pathways. These brain-endocast structure complexes are variable in size among birds, but the relationship between any brain structure and its endocast counterpart is unknown. To assess this relation- 
ship, optic tectum and hyperpallium volumes of 23 extant avian taxa were compiled from the literature and collected from existing histological series. Endocasts of the same species were generated from CT scan data of their skulls, and the surface areas of the corresponding endocast structures, the optic lobe and Wulst, were measured using Avizo and Maya. For each species, the volumes of the brain structures were regressed on the surface areas of the endocast structures. These preliminary results indicate that there is a positive relationship between endocast structure surface area and brain structure volume in the studied vision-related structures. This relationship was then used to predict the volumes of these brain structures in five extinct avian taxa (Archaeopteryx, Dinornis sp., Lithornis, Presbyornis, and "Buteo" grangeri). The taxonomic sample will be expanded in the future to strengthen these relationships and further our understanding of the evolution of brains, sensory systems, and their potential functional implications in birds.

\section{Using 3D Surface Data to Reconstruct the Sulcal Morphology of Fossil and Extant Canids}

\author{
Glidden $1^{a}$, Manger $P R^{b}$, Dunn $\mathrm{RH}^{c}$, Spocter $M A^{a, c}$
}

aDepartment of Biomedical Sciences, lowa State University, Ames, IA, USA; 'bSchool of Anatomical Sciences, University of the Witwatersrand, Johannesburg, Republic of South Africa; 'Department of Anatomy, Des Moines University,

Des Moines, IA, USA

spocter@gmail.com

The Canidae are a highly diverse family of vertebrates consisting of three subfamilies the Hesperocyoninae and Borophaginae, and the only surviving lineage the Caninae, which include domestic dogs and their living relatives. While the sulcal anatomy of the domestic dog has been well described, that of the extinct subfamilies of the Canidae have not been extensively studied using modern imaging techniques. Using MRI scan data obtained from seven extant canid species as well as spatial data obtained from the endocasts of eight extinct canids, we provide preliminary 3-dimensional surface models of the major canid groups and discuss key sulcal landmarks and the changes that accompanied the evolutionary transitions in the Canidae. Our results demonstrate that three-dimensional surface models generated using the NextEngine 3D scanners provide good clarity and depth and provide an excellent opportunity to mine surface features for relevant changes in sulcal anatomy as we reconstruct the evolutionary history of the canid brain.

\section{Enlargement of Telencephalic-Cerebellar Pathways in Parrots: Convergent Evolution with Primates?}

\author{
Gutierrez-Ibanez C ${ }^{a}$, Iwaniuk AN ${ }^{b}$, Wylie DR ${ }^{a}$
}

aNeuroscience and Mental Health Institute, University of Alberta, Edmonton, $A B$, and beuroscience, University of Lethbridge, Lethbridge, $A B$, Canada

cgutierr@ualberta.ca

Traditionally, the evolution of complex cognitive abilities has been associated with changes in the size of the telencephalon in general, or parts of the telencephalon, such as the prefrontal cortex in mammals or the nidopallium in birds. In mammals, increasing evidence suggests that changes in the size of the cerebellum, and areas that connect the cerebellum to the telencephalon are also related to the evolution of complex cognitive abilities. In humans and other primates, there is an expansion of cortico-ponto-cerebellar pathways, including a closed loop circuit between the prefrontal cortex, the pontine nuclei and the cerebellar hemispheres. Some birds, particularly parrots and corvids, (1) have a relatively large telencephalon, similar in size to primates, and (2) exhibit complex cognitive abilities that rival those in primates. Comparative studies suggest that changes in the size and complexity of the cerebellum in birds are associated with complex behaviors, such as tool use, but it is not known if this also involves changes in the size of structures that connect the telencephalon and the cerebellum. Birds might differ from mammals given that telencephalo-pontocerebellar pathways are less well developed. In birds two nuclei at the base of the pons, the medial and lateral pontine nuclei (PM and $\mathrm{PL}$, respectively) send mossy fiber projections to the cerebellum and receive projections from the telencephalon and have been proposed as homologues of the pontine nuclei of mammals. Birds have an additional nucleus that connects the telencephalon with the cerebellum: the medial spiriform nucleus (Spm). Spm is unique in that it lies in the thalamus rather than the pons and no homologue has been identified in mammals or other amniotes. Here we use a modern comparative approach to examine the relative size of the pontine nuclei, Spm, the telencephalon, the cerebellum and several visual regions that connect with the pontine nuclei, telencephalon and cerebellum across a wide variety of birds (100 species, 16 orders). First, we found that Spm, but not the pontine nuclei or other nuclei that connect the telencephalon with the cerebellum, are enlarged in parrots. Second, the relative size of the telencephalon and Spm are strongly correlated with one another. Finally, the relative size of the medial pontine nuclei is significantly correlated with that of several visual areas. We propose that the enlargement of Spm in parrots is associated with several factors: the enlargement of the associative pallium, the high density of neurons in the pallium, and finally a higher proportion of pallial to cerebellar cells. The enlargement of Spm in parrots suggests that the telencephalon-Spm-cerebellar pathway of birds may play an analogous role to cortico-ponto-cerebellar pathways of mammals, namely motor control and complex cognitive processes. Our results also indicate that the brains of parrots are rather unique among birds, and point to the Spm as a prime target for understanding the role of telencephalon-cerebellar pathways in evolution of complex cognitive abilities in birds. 


\section{Scaling of Thalamic Nuclei in Primates, Rodents and Carnivores}

Halley $A C^{a}$, Baldwin MKL ${ }^{a}$, Sherman $S M^{b}$, Krubitzer $L^{a, c}$

${ }^{a}$ Center for Neuroscience, University of California Davis, Davis, CA, bepartment of Neurobiology, University of

Chicago, Chicago, IL, and 'Department of Psychology,

University of California Davis, Davis, CA, USA

achalley@ucdavis.edu

Mammalian species are remarkably variable both in absolute brain size, and in the relative size of different brain areas. Differences in the allometric scaling of brain areas helps to distinguish particular lineages from one another, and suggest shared evolutionary adaptations and developmental programs. This is particularly true of sensory systems, as species differ widely in both the sensory periphery and associated sensory-mediated behavior. Sensory nuclei in the thalamus have been extensively studied in relation to visual system evolution and variation (e.g. dLGN), but limited data exists on the variation of other thalamic nuclei across species. Here we compare the relative size and organization of thalamic nuclei in a variety of species composed mostly of primates (e.g. titi monkey, rhesus macaque), carnivores (e.g. grizzly bear, cat), and rodents (e.g. ground squirrel, rat). Sectioned tissue stained for cytochrome oxidase, Nissl, AchE, VGLUT2, and/or myelin was used to delineate borders of both first-order sensory nuclei (e.g. dLGN, VPm/l, MGv) as well as higher-order nuclei (e.g. pulvinar, MGd). Threedimensional reconstructions of each thalamus were used to calculate individual nucleus volumes and to visualize the nuclear organization of individual species. Allometric analyses of individual nuclei are used to test for regular scaling patterns (concerted evolution) and deviations from them (mosaic evolution). Thalamic nuclei scale with absolute thalamus size at different rates, with positive allometry observed in certain areas (e.g. the medial dorsal nucleus, MD) and negative allometry in others (e.g. dLGN). We discuss these findings in relation to several major theories of brain area evolution, including both evolutionary and developmental arguments for allometric patterns between brain areas.

\section{Longevity Is Predicted by Absolute Number of Pallial Neurons, Not Body Size, Brain Size or Metabolic Rate, across Mammalian and Bird Species Alike}

\section{Herculano-Houzel S}

Depatment of Psychology, Department of Biological

Sciences, Vanderbilt Brain Institute, Vanderbilt University,

Nashville, TN, USA

suzana.herculano@vanderbilt.edu

Longevity has traditionally been associated with body mass across species, supposedly because of the decreased specific metabolic rate that is characteristic of larger animals. Recently, using the AnAge database (de Magalhães and Costa, 2009), GonzálezLagos et al. (2010) found that, after discounting the relationship with body mass, species of mammals with larger brains than ex- pected for their body size tend to live longer than those with smaller brains. The correlation is compatible with the cognitive buffer hypothesis that argues that large brains facilitate a longer lifespan. However, residual brain size only explained about $13 \%$ of the variance of residual lifespan across species.

Here I use the AnAge database combined with published data on brain mass for different species and (1) our own data on the numbers of neurons that compose different mammalian and bird brains ( $n=79$ species) or (2) estimates of numbers of pallial neurons in a much larger number of species $(n=705)$ in different clades of mammals and birds calculated from brain mass using clade-specific scaling rules that we have uncovered in the past. Analysis of dataset (2) shows that maximal longevity for each species varies as clade-specific functions of body mass, brain mass and metabolic rate. In contrast, longevity varies as a single, universal power function of the absolute number of neurons in the pallium of all mammal and bird species alike, with a joint exponent of 0.402 $\left(r^{2}=0.740, p<0.0001\right)$. For comparison, longevity can be estimated as a significant power function of body mass across mammal and bird species with a joint exponent of $0.133(p<0.0001)$, but a much lower $r^{2}$ value of 0.412 . Importantly, principal component analysis confirms that longevity clusters with number of pallial neurons, not with brain mass, body mass or metabolic rate. Age of female sexual maturity can also be predicted as a single power function of absolute number of pallial neurons across mammals and birds (exponent $0.471, r^{2}=0.652, p<0.0001$ ). Analysis of dataset (1) confirms that the absolute number of pallial neurons is the best predictor of maximal longevity and age at female sexual maturity compared to numbers of neurons in other brain structures, total number of brain neurons, brain mass, body mass and metabolic rate.

These findings indicate that the clade-specific correlations between maximal longevity and brain or body mass are best explained as a by-product of the universal correlation between number of pallial neurons and longevity in the face of the clade-specific correlations between numbers of pallial neurons and brain or body mass that we have uncovered in the past (Herculano-Houzel, 2017). That variations in longevity across mammalian and bird species alike are best explained by the sheer number of pallial neurons supports the view that larger numbers of pallial neurons offer a cognitive advantage that contributes significantly to extend the lifespan of a species. Longevity, which in this view should no longer be considered a by-product of metabolism, may thus offer a long sought-after global measure of cognitive success across species. 


\section{Many Selective Pressures Influence Brain Structure Simultaneously but Distinctively: An Examination of Patterns and Processes in Lizards}

Hoops $D^{a}$, Vidal-García $M^{a}$, Ullmann JFP, Janke $A L^{b}$, Stait-Gardner $T^{c}$, Duchêne $D A^{d}$, Price WSc Endler Jf, Whiting MJf, Keogh JSa

aDivision of Ecology and Evolution, Research School of Biology, The Australian National University, Canberra, ACT, ${ }^{b}$ Centre for Advanced Imaging, The University of Queensland, Brisbane, QLD, 'Nanoscale Organization and Dynamics Group, School of Science and Health, Western Sydney University, Sydney, NSW, dSchool of Life and Environmental Sciences, University of Sydney, Sydney, NSW, 'Centre for Integrative Ecology, School of Life and Environmental Sciences, Deakin University, Melbourne, VIC, and fDepartment of Biological Sciences, Discipline of Brain, Behaviour and Evolution, Macquarie University, Sydney, NSW, Australia daniel.hoops@mail.mcgill.ca

The brain plays a central role in a wide variety of functions such as behaviour, perception, motor control, and homeostasis. Each function undergoes different selective pressures over the course of evolution, and as selection acts on the outputs of brain function, it necessarily alters the structure of the brain. Two models have been proposed to explain the evolutionary patterns observed in brain morphology. The concerted brain evolution model posits that the brain evolves as a single unit and the evolution of different brain regions are coordinated. The mosaic brain evolution model posits that brain regions evolve independently of each other. It is now understood that both models are responsible for driving changes in brain morphology; however, it remains unclear what factors favour concerted or mosaic brain evolution. We have used lizards as a model system to examine concerted and mosaic brain evolution under a diverse suite of selective pressures. We find that concerted and mosaic brain evolution are occurring in unison, acting on the same brain structures in response to different selection pressures. We show that current neuroanatomy is the result of multiple selection pressures acting in different ways on the same structures. The impact of different selection pressures on a specific brain structure can be identified and separated, revealing the relative influences of difference selective pressures on evolutionary changes in brain structure. We find that though both models of evolution are occurring simultaneously in the same structures, but are only detectable when examining the particular drivers of selection. We show that the answer to the question of whether concerted or mosaic brain evolution is occurring in a particular system can depend more on the type of selection measured than the clade of animals studied.

\section{Measuring the Effects of Environmental Complexity on Telencephalon Volume Using High-Field Magnetic Resonance Imaging (MRI) in a Lizard Species}

LaDage $L D^{a}$, Kim $Y-E^{b}$, Neuberger $T^{c}$, , Lee $G^{d}$

aDivision of Mathematics \& Natural Sciences, Penn State

Altoona, Altoona, PA, bepartment of Engineering Science and

Mechanics, Penn State University, University Park, PA,

'Huck Institutes of the Life Sciences, Penn State University,

University Park, PA, and d Department of Bioengineering,

Penn State University, University Park, PA, USA

Idl18@psu.edu

Cognition is important in many ecologically-relevant behaviors and individuals that experience increased demands on their cognitive load also have larger telencephalon. One particular facet of the environment that can induce differential demands on cognitive processing is environmental complexity. Environmental complexity is composed of a variety of factors including increased spatial area use, spatial complexity, and number of conspecifics present, all of which can increase demands on cognitive processing. In particular, an increase in the complexity of an environment is related to an increase in spatial processing demands, which is reflected in the neural substrate responsible for processing spatial information. While clinical research has produced strong evidence that increased complexity of the environment can upregulate telencephalon attributes, likely due to the necessity of increased cognition in a more complex environment, clinical research may not always reflect what occurs in wild animals, particularly in species that exhibit much higher rates of brain plasticity when compared with mammals. Thus, the goal of the current study was to (1) ascertain the effects of environmental complexity on telencephalon volume in eastern fence lizards (Sceloporus undulatus) and (2) determine telencephalon volume at two different time points using Magnetic Resonance Imaging (MRI). Subjects were housed in standard laboratory conditions for 6 mo then subjected to a baseline MRI scan. After, lizards were transferred to a more complex environment, including increased overall spatial area and increased number of objects and hides within the environment; further, subjects were allowed social interactions with a conspecific. After six weeks, individuals underwent a second MRI scan. Our preliminary data indicate that, when subjected to increased environmental complexity, lizards tend to upregulate telencephalon volume compared with baseline MRI scans. Thus, it appears that lizards also respond to changes in environmental complexity by upregulating telencephalon volume. Importantly, this study also demonstrates that MRI can be leveraged as a powerful tool for non-invasively ascertaining brain plasticity over time in lizards and other non-model species. 


\section{Evolution of High-Acuity Circuitry in the Primate Primary Visual Cortex}

Miller DJ, Kaas $\mathrm{JH}$

Department of Psychology, Vanderbilt University, Nashville, TN, USA

daniel.j.miller@Vanderbilt.Edu

The cellular composition of functional modules within the brain provides crucial evidence to infer the patterns in evolutionary neuroscience that facilitate our understanding of the relationship between brain structure and function. In the primate visual system, information from the retina is routed through the thalamus and on to the cerebral cortex, with high-acuity vision from the fovea passing through the magnocellular and parvocellular streams of the thalamus to neurons in layer four (Layer 4A, 4B of Hassler, 1967; or Brodmann's 4C alpha, beta) of the primary visual cortical field (V1). Previous studies of the primate visual system suggest largescale phylogenetic trends in cortical organization as well as differences between diurnal and nocturnal species related to visual acuity. However, the systematic analysis of homologous circuits across multiple taxa using rigorous quantitative measurements are difficult because of a variety of logistical and methodological constraints. In this talk, I will first outline the strategy by which we have overcome some of these constraints by augmenting stereology with basic image segmentation and statistical learning procedures, and then discuss our results measuring the volume and counting the number of cells across the cortical mantle compared to layer 4 in $\mathrm{V} 1$ across primates (human, chimpanzee, baboon, macaque, squirrel monkey, owl monkey, tamarin, marmoset, galago). We used a combination of variation in cellular morphology, patterns of connectivity, enzymatic activity, and molecular gradients to define V1 from other cortical fields, as well as layer 4 within the cortical mantle, and counted cells in sections stained for Nissl or an antibody to neuronal nuclei (NeuN). Our results indicate a mosaic of phylogenetic shifts in the volume and cellular composition of the primate visual system associated with behavioral foraging ecology, suggesting the presence of selection pressures acting on this index of the computational capacity of cortical circuitry.

\section{What Is the Natural Biological Function of Dopamine in the Peripheral Auditory System and Does the Plainfin Midshipman Fish Have an Answer?}

\author{
Perelmuter $J T^{a, b}$, Sisneros $J A^{c}$, Forlano $P M^{a, b, d}$ \\ a'Behavioral \& Cognitive Neuroscience, City University of New \\ York (CUNY) Graduate Center, New York, NY, 'b Department of \\ Biology, CUNY Brooklyn College, Brooklyn, NY, 'Department \\ of Psychology, University of Washington, Seattle, WA, and \\ dEcology, Evolutionary Biology and Behavior, CUNY Graduate \\ Center, New York, NY, USA \\ jperelmuter@gradcenter.cuny.edu
}

Among its many functions in the nervous system, dopamine is thought to be a conserved modulator of the motivational salience of environmental stimuli. While dopaminergic modulation of au- ditory processing and motivational salience has been studied in the central nervous system, such modulation could also occur in the periphery, in the inner ear. The majority of investigations of dopamine in the ear use rodents as a model system, and focus on its inhibitory effect on primary auditory afferents, although there is some evidence for modulation of outer hair cells. The prevailing hypothesis put forth in these studies is that this inhibition serves to reduce damage to afferent neurons resulting from noise trauma. However, the stimuli employed in these experimental paradigms are louder and longer in duration than those likely to be encountered in the natural soundscape in which hearing systems evolved. A biological function for dopamine in the inner ear in the context of natural behaviors remains to be demonstrated.

Our work with the plainfin midshipman fish, Porichthys notatus, indicates that inner ear dopamine signaling is important for acoustic communication in social and reproductive contexts. Midshipman fish coordinate spawning activity in the intertidal zone in the late spring and summer. Female hearing sensitivity is enhanced throughout this period, an adaptation which is thought to help them locate mates at night via a male generated courtship call. Importantly, dopamine innervation is reduced in the inner ear and increased in the cholinergic auditory efferent hindbrain nucleus during the breeding season. We found that a single nucleus in the diencephalon projects to both the saccule, the main endorgan of hearing in midshipman, and the auditory efferent hindbrain nucleus. We characterized the ultrastructure of dopamine innervation and found direct synapses on cholinergic auditory efferent neurons in the hindbrain nucleus. In contrast, terminals do not form synapses in the saccule, indicating paracrine release and the potential for dopamine to modulate hair cells directly. We confirmed this with pharmacological manipulations of the dopamine system in the saccule with sound-evoked extracellular recordings from hair cells. Dopamine increases thresholds, and therefore appears to have an inhibitory effect on sound-evoked hair cell activity. Our results suggest that seasonal reduction of dopamine in the saccule serves as a release of inhibition to improve female hearing sensitivity. We propose that a novel biological function for dopamine in the peripheral auditory system is modulation of socialacoustic signals, a function which may be conserved in other vocal vertebrate species.

\section{Rampant Convergence between Mammals and Birds in Body and Brain}

Striedter $\mathrm{FF}^{a}$, Northcutt $R \mathrm{G}^{b}$

aDepartment of Neurobiology \& Behavior, University of California, Irvine, CA, and bepartment of Neurosciences, University of California, San Diego, CA, USA

georg.striedter@gmail.com

Mammals and birds have independently evolved a large number of similarities. For instance, they have independently evolved the ability to generate internal body heat. The evolution of endothermy was associated with turbinates inside the nasal cavity that help to retain body heat, with a significant reduction in body size, and with the evolution of effective thermal insulation (hair or feathers). Birds and other archosaurs also resemble most mam- 
mals in having evolved more efficient breathing and a parasagittal gait, rather than the sprawling type of locomotion seen in living lizards, turtles, and salamanders. On the sensory side, archosaurs and mammals have independently evolved tympanic ears, long cochleas, and specialized hair cells that allow them to hear high-frequency airborne sounds. Diurnal primates further resemble birds in having evolved a retinal fovea and excellent color vision, two features that early mammals had lost. Relative brain size is much larger in mammals and birds than in non-avian reptiles, and only birds and mammals seem to have a "prefrontal cortex." Particularly intriguing is that owls have a "visual Wulst" that is very similar to the primary visual cortex of mammals. However, many of the similarities between these structures probably resulted from convergent evolution, because the Wulst homolog in non-avian reptiles does not share them. In general, our analysis suggests that many features of mammalian neocortex are innovations that arose in stem mammals, even though some similar features evolved also in birds.

\section{Comparative Neuroanatomy of Navigational Maps in Primates}

Vanier $D R^{a}$, Schilder $B M^{b}$, Sherwood $C C^{b}$, Smaers $J B^{c}$

anterdepartmental Doctoral Program in Anthropological

Sciences, Stony Brook University, Stony Brook, NY,

${ }^{b}$ Center for the Advanced Study of Human Paleobiology,

The George Washington University, Washington DC, and

'Department of Anthropology, Stony Brook University,

Stony Brook, NY, USA

david.vanier@stonybrook.edu

A shift to a more sparsely distributed but energy-rich diet has been proposed as a driver of primate brain expansion, and increased spatial information processing for navigation is seen as central to this transition. The neurobiological underpinnings of spatial information processing for navigation in mammals are primarily located in subregions of the hippocampal formation (HP subregions). Here we investigate the evolutionary history of size changes in CA1, CA2, CA3, fascia dentata, hilus, subiculum, and entorhinal cortex from 43 primate species by scaling HP subregion volumes against the medulla. The time a brain region spends in development is believed to affect the size of that brain region in the adult organism. As the brainstem is the first segment of the neural tube to unfold during development, scaling HP subregions against the medulla allows the comparison of later-developing structures with an earlier-developing structure. Such a comparison is indicative of species differences in selective investment in specific HP subregions. Strepsirrhines have a larger relative entorhinal cortex, subiculum, and fascia dentata compared to anthropoids $(\mathrm{F}=8.682$, $p=0.005$ for the entorhinal cortex), while humans deviate from the anthropoid trend and show a strong increase in the relative size of their entorhinal cortex, subiculum, and CA3 subregions ( $\mathrm{F}=$ $11.619, p=0.002$ for the entorhinal cortex). The observed decrease in relative hippocampal formation subregion sizes in anthropoids relative to strepsirrhines occurs despite an increase in the relative size of the neocortex in anthropoids. Our results suggest that, com- pared to strepsirrhines, anthropoid brain evolution is characterized by a decrease in domain-specific investment for navigation in HP subregions amidst a domain-general investment in the neocortex. In humans, the very same hippocampal formation networks used in navigation are also activated during prospection, which plays a central role in our cognition according to an emerging body of evidence. The exceptional evolutionary expansion of human HP subregions observed here, together with recent evidence for the exceptional expansion of neocortical association areas in humans, suggests a joint selective expansion in humans of a neural system that supports mental time travel.

\section{Quantitative Analysis of Neural Cells in Different Structures of the Murine Brain Reveals that the Energetic Intake Is Constrained by the Neuronal Density of the Tissue}

Ventura-Antunes $L^{b}$, Botelho $L^{a}$, Tenorio $F^{a}$, Ricardo $M^{a}$, Gomes $G^{a}$, Herculano-Houzel $S^{b}$

anstituto de Ciências Biomédicas, Federal University of Rio de Janeiro, Rio de Janeiro, Brazil; bepartment of Psychology, Vanderbilt University, Nashville, TN, USA

lissa.ventura.antunes@vanderbilt.edu

Neuronal size, which can be gauged from neuronal density, is highly variable across species and structures. In contrast, the density of non-neuronal cells (glial and endothelial) is almost invariable, indicating that it is conserved throughout the evolution of mammals.

The brain is the tissue that consumes the most energy. However, it has been estimated that the microvasculature, formed by endothelial cells, occupies only about $2-4 \%$ of the cortical gray matter volume in the mouse. The small relative volume of the brain microvasculature raises the issue of how the tissue is organized metabolically, with the possibility of strong constraints imposed by this small vascular proportion.

Larger neurons are believed to cost more energy, at the very least because of the larger membrane surface area to repolarize. However, our estimates of how brain metabolic cost scales with numbers of neurons across species suggest that larger neurons do not cost more energy. Here we set out to investigate whether across brain regions within a single species, the mouse, those with lower neuronal densities (and thus larger neurons) are associated with proportionately larger capillary densities, which would maintain a constant amount of blood available per neuron across brain structures. This expectation is based on the finding that blood flow and glucose consumption, which are linearly proportional across mouse brain structures, also have a direct and linear correlation with capillary density (Klein et al., 1986). Thus, variations in vascular density across structures are proportional to variations in glucose use.

We used systematic random sampling of 3D image stacks and 2D microscopic images of regions of interest across various structures of the brain of 5 adult mice. While analyzing structures in three dimensions is more accurate, it is very time-consuming, and 
here we find that two-dimensional images allow us to gather more data in less time with similar results. We find that, as expected, glial cells are more common than endothelial cells (in an average proportion of 70:30 among non-neuronal cells). Also as expected, the microvascular fraction is higher in the cortical gray matter cortex than in white matter cortex and granular structures. Contrary to our expectations, however, we found that ratio of endothelial cells per neuron decreases with increasing neuronal density in an inverse relationship that is remarkably uniform across brain sites, which indicates that larger neurons have more energy available to them. Our results suggest that it is not necessarily that larger neurons within an individual brain require more energy, but rather that, because capillary density does not accompany neuronal density fast enough, it follows that smaller, denser neurons are limited in the amount of energy they can get.

\section{Tonotopy and Time Difference Sensitivity in Turtles} Willis $K L^{a, b}$, Carr $C E^{a}$

aDepartment of Biology, University of Maryland, College Park, MD, and ${ }^{\text {b}}$ Department of Biology, University of Oklahoma, Norman, OK, USA

kwillis@ou.edu

The hearing range of turtles is about $50-1000 \mathrm{~Hz}$, with thresholds above $50 \mathrm{~dB}$ SPL. Compared to other reptiles, turtles hear much lower frequencies and have much higher thresholds, possibly associated with hearing both in air and in water. Hearing limited to low frequencies can constrain sound localization abilities, particularly in red eared slider turtles, which have a small head, isolated middle ears, and an amphibious lifestyle. To determine if the turtle's auditory system was sensitive to interaural time differences (ITDs), we investigated the physiological responses of the brain stem auditory nuclei, nucleus magnocellularis (NM) and NL.

Recordings of single units in NM and NL to dichotic sound in an isolated head preparation revealed best frequency responses from $100-600 \mathrm{~Hz}$, with thresholds from 60-80 dB SPL. NM neurons phase locked reliably, and projected bilaterally to NL, which was sensitive to ITDs. Measures of characteristic delay revealed best ITDs around $\pm 200 \mu \mathrm{s}$. Turtle NL neurons typically also had characteristic phases close to 0 , consistent with binaural excitation. Thus turtles encode ITDs within their physiological range.

Additionally, we combined anatomical and physiological techniques to investigate a second key feature of auditory systems; tonotopic organization. Using neurobiotin to fill the auditory branch of the VIII nerve, we found both bouton and en passant nerve terminals on to NM neurons but no endbulb terminals. The turtle basilar papilla is tonotopically organized, but neither small targeted injections of neurobiotin, nor physiological mapping within NM, showed any tonotopic organization. Thus the low frequency hearing range of turtles may not be associated with tonotopic organization in brain. 